\title{
Article
}

\section{University Social Responsibility: The Case of Italy}

\author{
Maurizio Comoli ${ }^{1}$, Lorenzo Gelmini ${ }^{1}$, Valentina Minutiello ${ }^{2, *(D)}$ and Patrizia Tettamanzi ${ }^{2}$ (D) \\ 1 Department of Economics and Business Studies, University of Eastern Piedmont, 28100 Novara, Italy; \\ maurizio.comoli@uniupo.it (M.C.); lorenzo.gelmini@uniupo.it (L.G.) \\ 2 School of Economics, Cattaneo University (LIUC), 21053 Castellanza, Italy; ptettamanzi@liuc.it \\ * Correspondence: vminutiello@liuc.it
}

\section{check for}

updates

Citation: Comoli, Maurizio, Lorenzo Gelmini, Valentina Minutiello, and Patrizia Tettamanzi. 2021. University Social Responsibility: The Case of Italy. Administrative Sciences 11: 124. https://doi.org/10.3390/admsci 11040124

Received: 16 August 2021

Accepted: 25 October 2021

Published: 29 October 2021

Publisher's Note: MDPI stays neutral with regard to jurisdictional claims in published maps and institutional affiliations.

Copyright: (c) 2021 by the authors. Licensee MDPI, Basel, Switzerland. This article is an open access article distributed under the terms and conditions of the Creative Commons Attribution (CC BY) license (https:// creativecommons.org/licenses/by/ $4.0 /)$.

\begin{abstract}
Increasing attention is now being paid to the concept of sustainability as a crucial element of our life at all levels. The awareness that attention must be paid not only to the present, but also and above all to the future of the society in which we live has increased attention to social and environmental issues, such as climate change and the digital revolution. This transformation has also impacted the public sector: in particular, the scientific attention in the university sector has led to the birth of the concept of University Social Responsibility (USR), which suggests that universities sustainably re-transform their work. However, this issue has so far only been the subject of a few studies. The purpose of this article is to promote greater awareness on the part of universities of the importance of addressing sustainability issues. The results of the analysis, obtained thanks to the use of a questionnaire and interviews, depict the state of the art in the adoption of social reporting practices by Italian universities and identify the main reasons and barriers to the adoption of these practices.
\end{abstract}

Keywords: university social responsibility; universities; social responsibility; CSR reporting; Italy

\section{Introduction}

The current context is drastically changing the way we live, with effects on events (especially natural ones) on a global scale as well as on our daily lives. Consequently, the issue of sustainability in this transformation process has assumed a crucial role, as it has become clear that the future of our society cannot be based solely on economic issues, but will have to face continuous and pressing cultural and climatic, geopolitical and technological evolutions (Galantino 2017).

Initially focused on sustainability within the business world, the literature has progressively extended research to the public sector and university institutions, through the introduction of the topic of University Social Responsibility (USR) (Lee 2000; Wright 2002; Cortese 2003; Rusconi 2005; Velazquez et al. 2006; Fornasa and Salomone 2007; Albrecht et al. 2007; Frey 2009; Del Sordo et al. 2010; Jones et al. 2010; Lozano 2011; ASEAN University Network (AUN) (ASEAN University Network (AUN)) 2012; Lozano et al. 2013; Romolini 2015; Bokhari 2017).

This new paradigm has led universities to redefine their mission and functions, implementing a process of continuous change in which the traditional "missions" of training and research are reproposed in a sustainable and value creation perspective (Gulbrandsen and Slipersaeter 2007). In this way, universities can respond to the increasingly pressing expectations of their stakeholders, thus reacquiring and consolidating the relationship of trust between civil society and the university system (Buzby and Falk 1979; Moggi 2016). In this scenario, characterized by greater complexity and dynamism, the task of the universities was to review their governance structures, performance measurement and reporting, providing an adequate response to the growing and pressing information needs of their social interlocutors, as well as to become more aware of the deeper meaning of their missions and the social/environmental impact of their actions. 
This paper aims to promote greater awareness on the part of universities of the relevance of this subject. It represents a starting point for further study on this underinvestigated research topic. The paper aims to answer two research questions, one consequent to the other. The first, concerning the analysis of the characteristics of the Italian academic panorama about social reporting practices, highlighted a very fragmented situation, requiring as a logical consequence the definition of a second research question, relating to the motivations and barriers underlying development and dissemination of social reporting practices. Thanks to the use of questionnaires and interviews, a clearer picture of the current state of social reporting of Italian universities was obtained, highlighting the development and the constraints that hinder its full adoption.

The structure of the paper is as follows. Section 2 is dedicated to the review of the literature on the topic of CSR within the academic world, on the definition of USR and the different forms of social reporting of Italian universities and the current level of dissemination. Section 3 describes the sample selection and methodology. Section 4 presents the main results. Finally, the implications and limitations of the work are discussed and an agenda for future research is provided.

\section{Literature Review}

\subsection{Setting the Scenario: A Diachronic Analysis of the Social Reporting Made by Universities}

The objective of the analysis is to present the social reporting practices carried out within the national academic landscape, to underline the prevailing characteristics and the practices implemented.

As currently in Italy there is no database containing all the social reporting tools and related models adopted by each university, all the information found on the MIUR website (and sites connected to it) has been included for the search of the documentation.

From the MIUR census relating to the period 2017/2018, it emerged that the national academic landscape is made up of 98 universities divided into public and private universities (11 of which are legally recognized as telematic) as proposed in Figure 1.

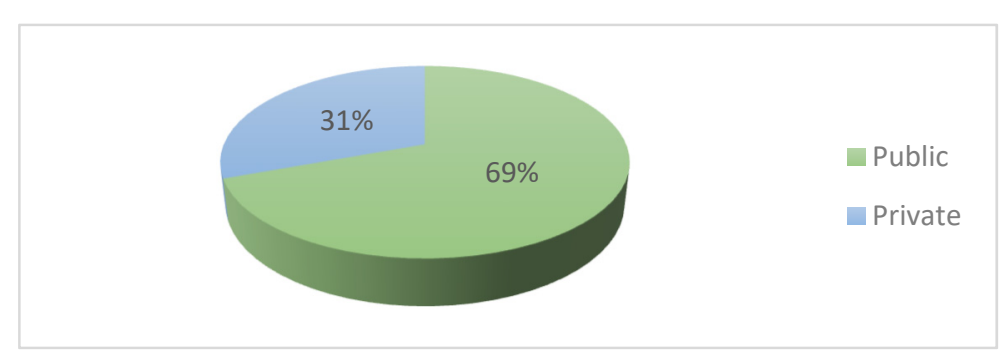

Figure 1. Type of university (private or public) on the Italian scene in the academic year 2017-2018.

To give greater relevance to this first data, the geographical distribution of the universities was taken into consideration: using the subdivision proposed by ISTAT of the Italian regions into four macro-areas, the predominance of public universities compared to private ones is confirmed at the national level (Figure 2).

The MIUR statistics referring to the academic year 2017/2018 include only 90 of the 98 universities and private universities are mainly small in size, while medium and mega universities are predominantly, if not exclusively, of a public nature (Figure 3).

Specifically, it is noted that most of the public universities with more than 40,000 members are located in the north. The medium-sized universities are mainly of a public nature (19 against 4 of a private nature) and are located for the most part in Northern and Southern Italy. Conversely, private institutions ( 23 universities) tend to be small and are mostly located in Central Italy. 


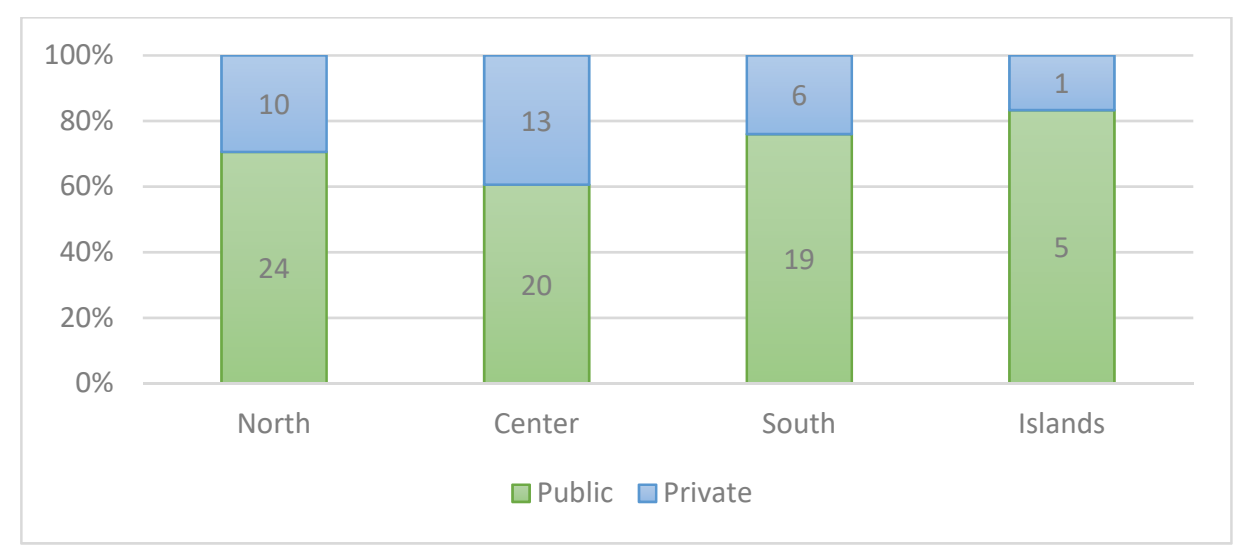

Figure 2. Geographical distribution, in Italy, based on the nature of the University.

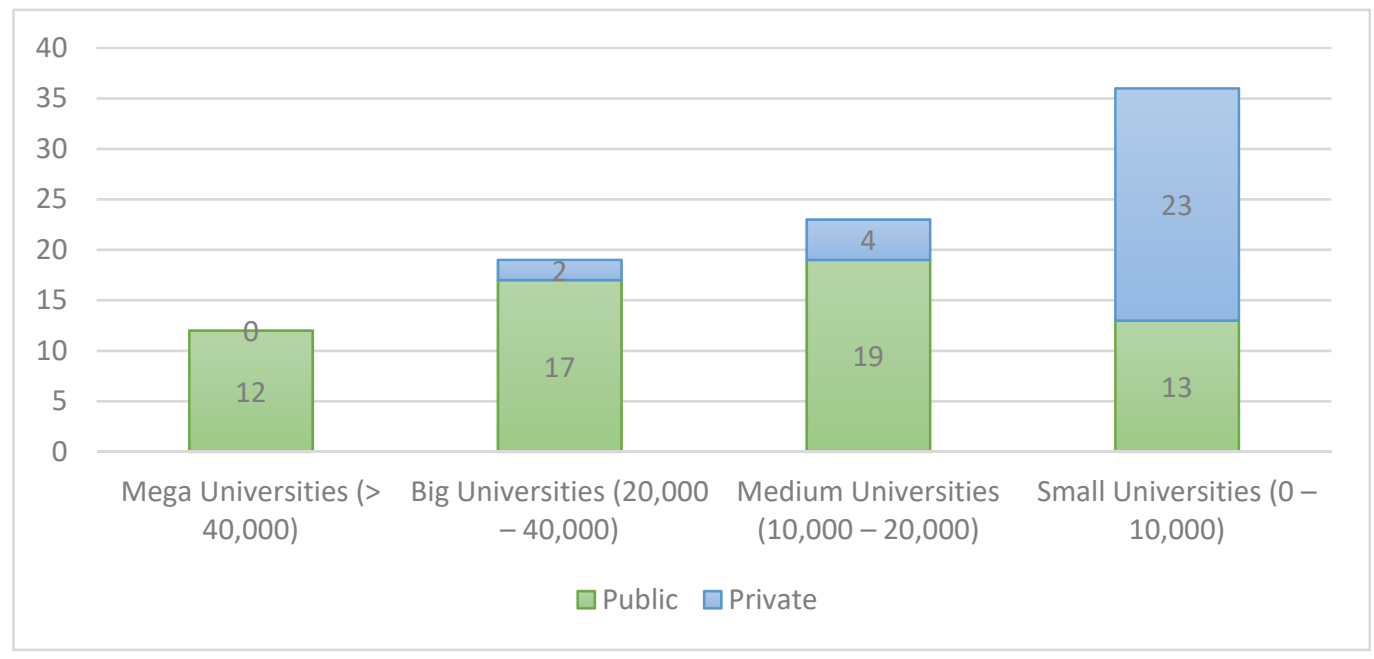

Figure 3. Size of Italian universities in relation to their nature.

\subsection{The Concept of Accountability in the Context of Italian Universities}

Just like businesses, universities are also social institutions aimed at creating and sharing value with multiple stakeholders; thus, their ability to express their social and cultural function becomes a sine qua non condition (the so-called "third mission"), as well as helping to clarify the impact of their policies, actions, decisions and performance through reporting processes that follow the same guidelines and accountability standards adopted in the business world (GBS 2016).

In the book Sustainability Reporting in Universities, Moggi (2016), offered a brief but meaningful study of the reasons that push universities to promote a social reporting process. Some of the reasons underlying the implementation of a reporting system concern:

- The holistic representation of the university's results in qualitative and quantitative terms, highlighting the resources used and the activities carried out to pursue the academic mission;

- The desire of the university to begin a sustainability process by communicating its work to stakeholders and by assessing its economic, social and environmental impacts within the community and the territory of reference. However, this objective cannot be separated from a preliminary research phase in which knowledge about sustainability is acquired, consolidated and increased;

- The intention to adopt reporting tools designed to improve public relations, supporting the image of the university in the context in which it operates by describing the use of resources (including the public ones) received; this "self-referential" purpose, 
although not always in line with the editorial standards indicated in the main reference frameworks, can represent a vehicle for increasing the competitive advantage over other universities that do not adopt this tool;

- The desire to "follow" the path of universities that have already undertaken this process following the growing pressure from the institutional context (Moggi 2016).

By focusing on the Italian context, the literature has shown that Italian universities tend to lag on the issue of social reporting compared to international ones. However, in recent years, a process of significant change has been initiated thanks to the creation promoted by the CRUI (Conference of Rectors of Italian Universities) of the "University Network for Sustainability" (RUS). Established in 2015, the RUS represents the first experience of coordination and sharing between all Italian universities involved in the issues of environmental and social responsibility. It defines specific groups of work on issues considered strategic and transversal to the achievement of its institutional objectives: climate change, education, energy, mobility and waste.

Among the main aims promoted by the RUS are:

- The dissemination of a common culture about sustainability through the sharing of best practices, knowledge and multidisciplinary skills to increase the positive impacts implemented by each university (but also applicable to other sectors of the $\mathrm{PA}$, education and the territory in general);

- The promotion and achievement of the Sustainable Development Goals (SDGs);

- The strengthening of the value and recognition of the Italian experience on the international scene. Thanks to participation in a network of common values and metrics, it is possible to "mitigate" the isolation of Italian universities within international rankings due to specificities not found in other academic contexts.

The RUS is open to all universities belonging to the CRUI and to other non-profit organizations whose institutional objectives are in line with those promoted by the network. In this way, through the appointment of a referring delegate for the network, each University and organization can share its sustainability knowledge and practices (RUS 2019).

\subsection{The Social Reporting Carried Out by the Universities}

It should be highlighted that the meaning of social reporting is attributable to both sustainability and social and environmental reporting. These denominations are commonly recognized as social accounting practices (Moggi 2016).

The main forms of social reporting that are most widespread on the national academic panorama are proposed below. However, given the substantial freedom granted to universities to adopt the most functional reporting model for their communication and editorial needs, there has been (as within other forms of organization) a proliferation of documents with very different characteristics in terms of structure, content and form (GBS 2016).

\subsubsection{The Social Report}

Considered one of the first accountability tools aimed at communicating USR practices to social interlocutors, today it is among the most widely used forms of reporting on the national scene. For this reason, to ensure a correct qualitative and quantitative representation of the university reality, it is essential to define a drafting process that is as precise and rigorous as possible.

A first consideration to make is to underline that the social report does not aim to replace the financial statements (as an instrument of the university's capital, economic and financial representation) but intends to support it, adding information not available from the accounting tools.

In this perspective, the social report, therefore, takes on the function of reporting to its community of reference the impacts generated by the university at an environmental, social and economic level, always bearing in mind the multiple expectations of the relevant stakeholders. 


\subsubsection{The Mandate Report}

The mandate report has as its main objective the reporting of the results achieved on based on what was defined at the beginning of the period. It is easy to understand how the structure and content of the mandate report are profoundly different from those envisaged in the social report. The first difference to underline is the reference time frame, since while the first covers a multi-year period (whose duration varies according to the administrative mandate), the second tends to refer to the calendar year.

\subsubsection{The Environmental Report}

The purpose of the environmental report is to "provide an organic picture of the direct interrelationships between the company and the natural environment through the representation of qualitative and quantitative data of the environmental impact of production activities and the economic-financial effort sustained by the company for the protection of the environment" (Fossati et al. 2009). Unlike the social report which contemplates all the relationships with possible stakeholders, the report focuses on the ecological impacts of the activity carried out by the company.

Although there is still no ad hoc environmental management standard for public interest entities and universities, it is worth mentioning the model proposed by Dionisio (Moggi 2016) in which there is a subdivision into three levels of the environmental balance:

- The first level is almost qualitative in nature since, after an initial description of the activity carried out by the organization, all the information regarding the environmental impact generated by the company is detailed, also concerning the effects generated on employees and the community of reference. This section refers to any environmental certifications, also indicating the presence and evolution of an internal system designed specifically for environmental management;

- At the second level all the data (and related performance indicators) relating to the use of resources and the effects produced by the outputs on the environment in terms of waste produced, emissions into the atmosphere, noise pollution are reported;

- The last level is functional to the communication of the main integrated sustainability policies and strategies to be implemented to mitigate and prevent the environmental impacts caused by the organization's production process.

\subsubsection{The Gender Report}

In Italy, this type of report is recommended by the directive of the Department for Rights and Equal Opportunities of 2007, to promote a culture of public administrations aimed at sustaining the enhancement of contributions offered by men and women, reducing implicit and explicit obstacles and discrimination. With specific reference to universities, at the initiative of the CRUI, in 2018 the MIUR approved a document entitled "Indications for positive actions of the MIUR on gender issues in Universities and research" in which, drawing inspiration from the best practices already adopted at European level, the guidelines to encourage the equal presence at any hierarchical level were defined.

\subsubsection{The Mission Report}

The mission report can be considered as the social report of non-profit companies. It allows the organization to communicate effectively the mission pursued (not merely in economic terms), indicating the results achieved and the lines of action taken to be able to achieve them.

\subsubsection{The Sustainability Report}

The sustainability report allows organizations to assess the impact of non-profit issues. The last decade has seen a significant increase in the sustainability report within profitoriented organizations as, through the help of some transversal indicators, it represents the most transparent and adequate way to represent the performance achieved in terms of sustainability, values of the organization, governance model and link between the strategy 
and its commitment to promote a sustainable global economy. This trend has also managed to spread within the world of non-profit organizations, public interest entities and, in particular, within universities as, given the nature of the entity, the three dimensions of sustainability are closely related to their mission (GBS 2016).

In practice, the name of "Sustainability Report" is attributed to the guidelines and drafting schemes referable to the guidelines of the Global Reporting Initiative (GRI), a constantly updated framework which, as subsequently highlighted, is experiencing growing interest within all sectors.

\subsection{Recent Contributions in the Academic Field}

Even if the theme of USR is still in development amongst academics, some literature has already highlighted potential topics and issues.

According to some authors (del Mar Alonso-Almeida et al. 2015; Del Sordo et al. 2016) the diffusion of sustainability reporting is still at an early stage in universities, and in Italian as well, despite the increasing concerns about sustainability in young people and other universities' stakeholders. If the adoption of USR practices certainly fosters university visibility and facilitates the ability to raise funds for future sustainability activities, at the very same time the development of social reports has been challenged by the lack of systematic collection of non-financial information within the university.

Furthermore, in line with Larrán Jorge et al. (2019), sustainability disclosure practices by universities are explained by different factors, as institutionalization, geographical region, external assurance and leadership.

Quite interestingly, with specific reference to Italy, some authors have mentioned the importance of societal issues, as COVID-19, inside the corpus of web-based USR (Nicolò et al. 2021), and the relevance of intellectual capital disclosure and its components (Nicolò et al. 2021).

Meseguer-Sánchez et al. (2020), analyzed the scientific production of the economic and environmental impacts of USR over the last 50 years through a bibliometric analysis. According to them USR, as a "mechanism that allows the dissemination of HEI values and so ensures their economic, environmental, and social sustainability", is committed to the demands of its various interest groups.

According to Ali et al. (2020), "Universities across the globe need to undertake social responsibility as an integral process of the organization and mold their activities of teaching education and training accordingly"; in this sense, their research indicates the practical approaches that can be undertaken by universities to legitimize their social responsibility initiatives, also shedding light on the fact that the social responsibility of universities differs "in nature from corporate social responsibility since the nature of institutional operations and objectives are inherently different from business organizations".

Rababah et al. (2021) showed that "University management creativity, effective communication with the public and stakeholders, the quality of the educational process and the development of scientific activities stimulate USR development", with special regard to the BRICS countries and, as such, these should be used as the basis for the strategic planning of activities in the context of the continuing COVID-19 pandemic.

Another fruitful avenue of research, in the specific context of universities, is in the field of Green Public Procurement (GPP), which could, in turn, foster and improve university social responsibility and its disclosure.

If Cheng et al. (2018) have already performed a systematic literature review up to 2016 - showing that GPP discussion has mostly focused on the specific impacts of GPP implementation, while the discussion on GPP as compared to other environmental policy tools, in terms of efficiency and innovation, is still lagging-in 2021 two distinct streams of authors (Liu et al. 2021; Ma et al. 2021) demonstrated, with their findings, that external stakeholder drivers have a positive relationship with GPP practice. Both the knowledge of GPP implementation policies and the knowledge of GPP benefits mediate this relationship. 


\section{Methodology}

As anticipated in the introductory part, to carry out the research part it was decided to adopt a mixed-method, through the use of tools typical of quantitative and qualitative research, in order to guarantee both a better understanding of the data and to overcome weaknesses that distinguish the two methods.

Both analyses of universities' sites, documentary analysis, a survey and interviews have been developed.

\subsection{Universities' Sites and Documentary Analysis}

A preliminary analysis of the sites of the 98 universities was carried out to verify the presence of sections dedicated to sustainability containing practices implemented and social reporting documents published up to 10 February 2019.

In this first phase of documentary analysis, some criticalities were found regarding the availability of the reports published by the individual universities as they are not always easy to access. Specifically, the research (to be able to recover all documents) was conducted by consulting:

- The area of the university website dedicated to sustainability;

- The previous versions of the website and the historical archives available (in particular for the first published reports);

- Posters of conferences dedicated to the theme of sustainability in universities (where links to university documents are shown).

If unable to retrieve the report online, it was acquired through telephone contact with the competent office (generally the public relations office and the accounting and budget office).

From what is highlighted in these first passages, it is easy to understand that there is an underlying communication problem, as not all universities perceive reporting models as a communication tool towards their reference stakeholders (thus facilitating their accessibility).

\subsection{The Questionnaire: A Methodological Note}

Based on the objectives to be investigated, it was decided to use a questionnaire (typical of quantitative research) as it constitutes a valid measurement tool capable of obtaining an initial confirmation of the sensitivity regarding university policies about social responsibility within the individual universities of the sample. Consistently with the research objectives, the questionnaire was administered through the Google Forms platform to a sample made up of Italian universities belonging to the network of Universities for Sustainable Development (RUS) and the Study Group for Social Report (GBS). The objective was to investigate the level of sensitivity towards the practices of dissemination of USR policies, as well as to analyze possible reasons and factors that limit their adoption.

As most of the universities participating in the GBS also participate in the RUS, only the RUS contact for these universities was selected for simplicity's sake (for a matter of updating the contact list of the respective referents).

Sixty-four universities adhering to the RUS were identified according to the latest data updated on 26 February 2019, and four universities adhering only to the GBS. This assumption in the selection of the sample involves the coverage of about $70 \%$ of the total population.

Thanks to the data available on the RUS and GBS websites (although not completely updated), the e-mail addresses of the delegates of the rectors and/or of the operational contacts of the network were selected: if both were indicated, it was decided to send the questionnaire to the contact person operating. As regards the GBS, since only the name of the contact person is indicated on the site, we proceeded to search for the relevant mailing address within the respective university pages.

It must be emphasized that with the selection made, all universities that do not belong to the two organizations were implicitly excluded; this does not mean that they do 
not belong to other associations/networks or that they completely exclude sustainability policies from their processes.

It consists of a set of ten closed and semi-closed questions (Appendix A). While the first is structured in such a way as to admit only dichotomous answers (yes/no) and multiple answers (admitting more than one answer among those predisposed), the second is characterized by the "other" option, so they offer the recipient the possibility of specifying in greater detail the topic analyzed by the closed question.

The questionnaire, of an anonymous nature to guarantee an adequate level of truthfulness in its completion, was accompanied by a letter of presentation in which the objectives investigated, the methodological note and the terms of presentation were explained.

\subsection{The Interviews: A Methodological Note}

Subsequently, some peculiar aspects detected in some universities were investigated by carrying out semi-structured interviews (typical of qualitative research), as an additional support and control tool compared to the questionnaire.

The approach is in line with Husband (2020), who reflects, "the semi-structured interview offers additional depth to that supplied by questionnaire or fully structured interview by inviting dialogic exchange. In so engaging, the researcher is actively constructing knowledge in partnership with the respondent".

During the period of administration of the questionnaire, some critical aspects identified in some of the 98 Italian universities were investigated by interview.

The four selected universities, one of which is private, were listed by letters of the alphabet to maintain an adequate level of anonymity, free from any form of conditioning that could affect the truthfulness of the answers.

The selection of universities was made by trying to investigate two types of needs:

- To understand the reasons behind the implementation (or not) of forms of social reporting within each university;

- To analyze the personal perception regarding the reasons and barriers underlying their full or partial applicability.

The four universities A, B, C and D, although they present a strong involvement and dissemination on sustainability issues and practices, show singular attitudes concerning the forms of social reporting. In particular:

- University A presented only one form of social reporting in the decade 2008-2018;

- Universities B and C to date have not yet implemented any form of social reporting;

- University D presents the social report every two years and has recently introduced, upon completion, also the gender report.

Once the reference sample was clearly outlined, we proceeded to identify and contact the most "adequate" figures to understand the topics covered by the interview.

The interviews, carried out by telephone, were preceded by preparatory calls and/or emails to present and clarify the research work, simultaneously defining a formal "meeting" date. The interview was set up according to a semi-structured scheme: using the questionnaire administered online as a reference scheme, the attention was subsequently placed on the single critical elements of the university on the adoption (or not) of forms of social reporting and the related personal perception of the phenomenon through questions consequential to the answers provided.

\section{Empirical Analysis and Results}

Given the criticalities that emerged from the literature review, it was necessary to carry out an in-depth study aimed at a better understanding of the sensitivity of Universities regarding USR practices and the reasons and limits that hinder the development and dissemination of social reporting practices. 


\subsection{Universities' Sites and Documentary Analysis: The Dissemination of Social Reporting}

After having contextualized the Italian universities in relation to their type, geographic location and size, we proceed to the presentation of the level of diffusion of the social reporting models.

Below is a first summary of the level of dissemination of social reporting for the type of university (Table 1).

Table 1. Incidence of the reports published out of the total of public and private universities.

\begin{tabular}{cccc}
\hline Type of University & N. Universities & N. Report & $\%$ \\
\hline Public & 68 & 35 & $51.47 \%$ \\
Private & 30 & 3 & $10.00 \%$ \\
Total & 98 & 38 & $38.78 \%$ \\
\hline
\end{tabular}

The data collected shows significant differences depending on the nature of the University. Specifically, it is assumed that $38.78 \%$ of universities have, at least once since 2008 , faced an approach to social reporting. The incidence of reporting is profoundly different depending on the type of university, as only $7.89 \%$ of private universities have shown interest in this, even just once.

Going into the detail of the figure below, the distribution of the universities that adopt social reporting practices is presented for the geographical area of reference (Figure 4).

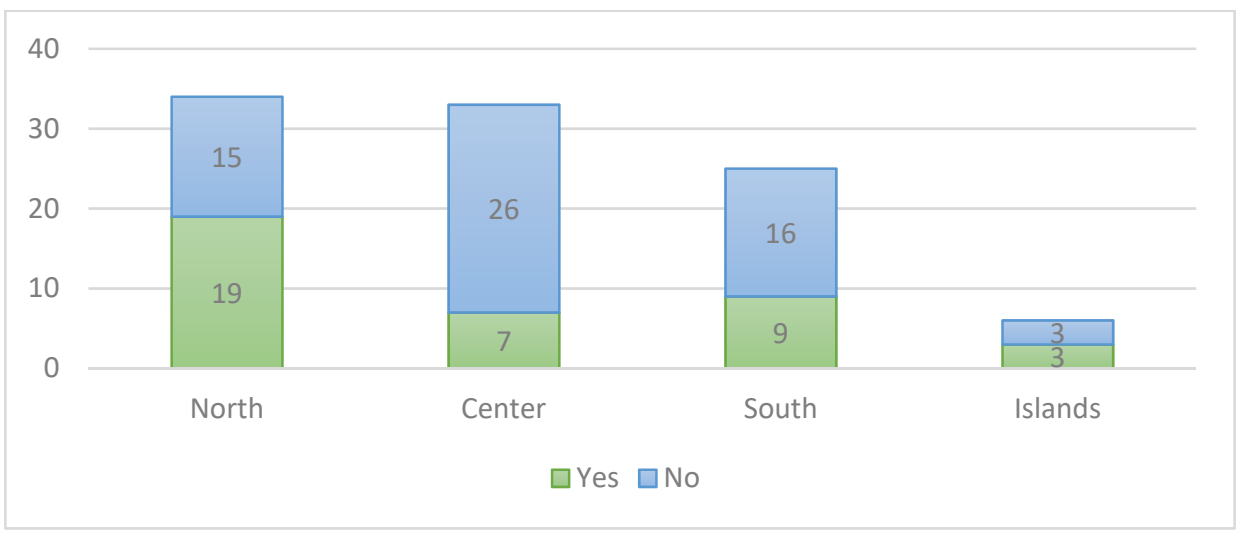

Figure 4. Dissemination of social reporting by geographical area.

The graph shows, both in absolute values and in relative terms (by comparing the reporting universities with the total number of universities in the geographical area), how the universities located in Northern Italy are more active in social reporting practices.

As for the Sicilian and Sardinian universities, even if there are only three reporting, when compared with the total number of universities in the area they represent $50 \%$ (Table 2).

Table 2. Dissemination of social reporting with respect to the presence of universities by geographical area.

\begin{tabular}{|ccccc|}
\hline Geographical Zone & Social Report & No Social Report & Total & $\%$ \\
\hline North & 19 & 15 & 34 & $55.88 \%$ \\
Center & 7 & 26 & 33 & $21.21 \%$ \\
South & 9 & 16 & 25 & $36 \%$ \\
Islands & 3 & 3 & 6 & $50 \%$ \\
Total & 38 & 60 & 98 & \\
\hline
\end{tabular}

A further critical element to consider in the survey is the correlation between the size of the university and its nature: the table below shows a greater sensitivity of mega and large/medium universities towards social reporting compared to small ones, which 
account for less than 14\%. As for the year 2017/2018 the MIUR does not provide the number of students enrolled for the University Institute of Advanced Studies of Pavia (IUSS), the last available data dating back to 2010, equal to 297, was taken as a reference.

This phenomenon has peculiar characteristics as it can be seen that, although the size of the university decreases and the number increases, there is no consequent increase in reporting universities (Table 3 ).

Table 3. Dissemination of social reporting by university size.

\begin{tabular}{cccccc}
\hline & & Social Report & No Social Report & Total & $\%$ \\
\hline Mega & $>40.000$ & 10 & 2 & 12 & $83.33 \%$ \\
Big & $20.000-40.000$ & 11 & 8 & 19 & $57.89 \%$ \\
Medium & $10.000-20.000$ & 12 & 11 & 23 & $52.17 \%$ \\
Small & $0-10.000$ & 5 & 31 & 36 & $13.89 \%$ \\
Total & & 38 & 52 & $90 *$ & \\
\hline
\end{tabular}

*As anticipated, the MIUR statistics referring to the academic year 2017/2018 include only 90 of the 98 universities.

\subsection{Time Analysis of the Phenomenon}

In the figure shown below, we aim to present the temporal evolution of the dissemination of social reporting models over the last 10 years, indicating the relative trend line. Although the analysis carried out does not presume to represent a complete evolutionary trend in the social reporting of universities, the following graph indicates, albeit with a fluctuating trend, a growing sensitivity to the issue by Italian universities.

The hypotheses underlying this phenomenon are attributable, for example, to the technical times of data collection rather than to physiological—organizational and internal governance changes (Moggi 2016).

Although the use of accountability tools is not yet integrated with the university's decision-making and management processes, it is interesting to underline a growing trend, reaching a peak in 2018 with 18 reporting universities and 23 published reports (Figure 5).

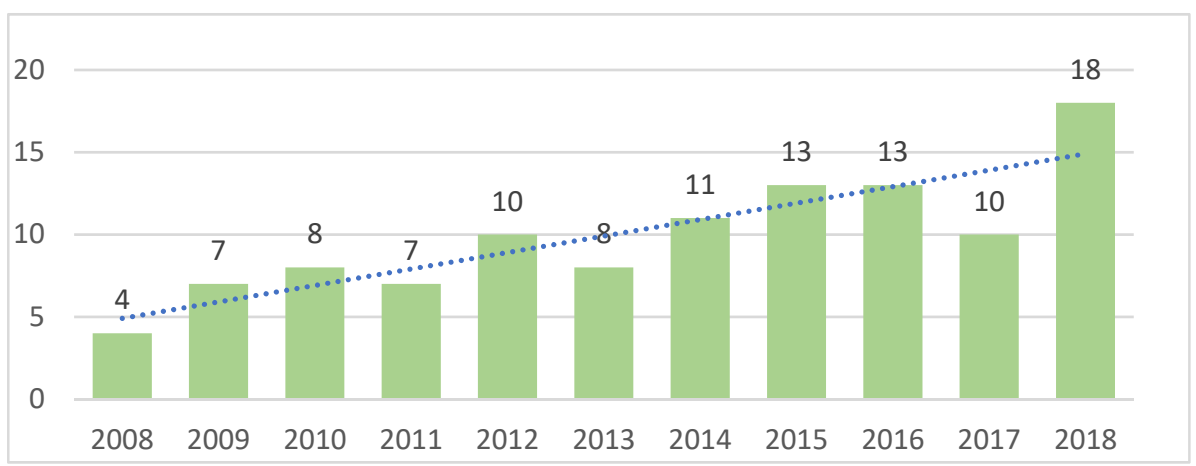

Figure 5. Time evolution in the publication of social reporting documents from 2008 to 2018.

Finally, we considered the reporting universities belonging to the university network and the study group for the social report. More than $50 \%$ of the members of the RUS and more than $63 \%$ of the GBS members implement a social reporting model and, consequently, we conclude that not all the members of the RUS and the GBS draw up reports and not all the reports are produced by universities belonging to the two organizations.

Entering into the merits of the 38 universities identified, we proceed to the representation of the 23 documents published in 2018. It is clear how the published reports are attributable to six macro-categories where the most significant weight is taken on by social and gender balance sheets $(34.78 \%)$, followed by the sustainability reports (Table 4 ). 
Table 4. Types of reporting for the year 2018.

\begin{tabular}{|ccc}
\hline Type of Reporting & N. Report & $\%$ \\
\hline Social report & 8 & $34.78 \%$ \\
Gender report & 8 & $34.78 \%$ \\
Sustainability report & 4 & $17.39 \%$ \\
Environmental report & 1 & $4.35 \%$ \\
Mission report & 1 & $4.35 \%$ \\
Mandate report & 1 & $4.35 \%$ \\
\hline Total & 23 & \\
\hline
\end{tabular}

\subsection{The Questionnaire}

Out of a total of 68 questionnaires administered from 21 March 2019 to 12 April 2019, 25 responses were received (approximately $37 \%$ of the total). The analysis presented below provides a grouping of the questions into macro-topics.

4.3.1. Formalized Policies and Substantial Commitment to University Social Responsibility Practices

There is a modest level of attention by universities to issues related to sustainability; specifically, $76 \%$ of universities declare that they adopt formalized procedures in favor of the USR, including:

- The use of an ad hoc register dedicated to its suppliers and related tools to evaluate their performance in qualitative-quantitative terms;

- A formalized management of waste collection and disposal (also through the involvement of staff and students) as well as structural and behavioral actions aimed at improving the energy efficiency of the university (monitoring the carbon footprint parameter) and sustainable mobility.

Although only slight reference to the topics is given within the university statute (only $44 \%$ of respondents have included explicit references), significant emphasis is given to sustainability in the context of the university's first mission: teaching.

On this issue, as many as $80 \%$ declare that they have included ad hoc training courses on sustainability issues; this figure represents an indicator of how sensitive and aware universities are of how the new generations can themselves be vectors of a process of change and promoters of sustainability within society.

This question, limiting the possibility of inserting references to sustainability only within the statute, is not suitable for completely intercepting the phenomenon observed. As a starting point for further research, it would be interesting, in the drafting phase, to broaden the scope by deepening analysis of whether or not there are further references within the code of ethics or internal regulations prepared by the university.

Although $60 \%$ of respondents have not yet set up a formalized organizational function within their organization chart, $56 \%$ state that they periodically allocate research funds from their university budget by defining ad hoc research projects, some of which are related to the achievement of defined objectives from the 2030 Agenda that the university has set itself to achieve, a sign of active interest and particular involvement on the part of professors and researchers (Figure 6). 


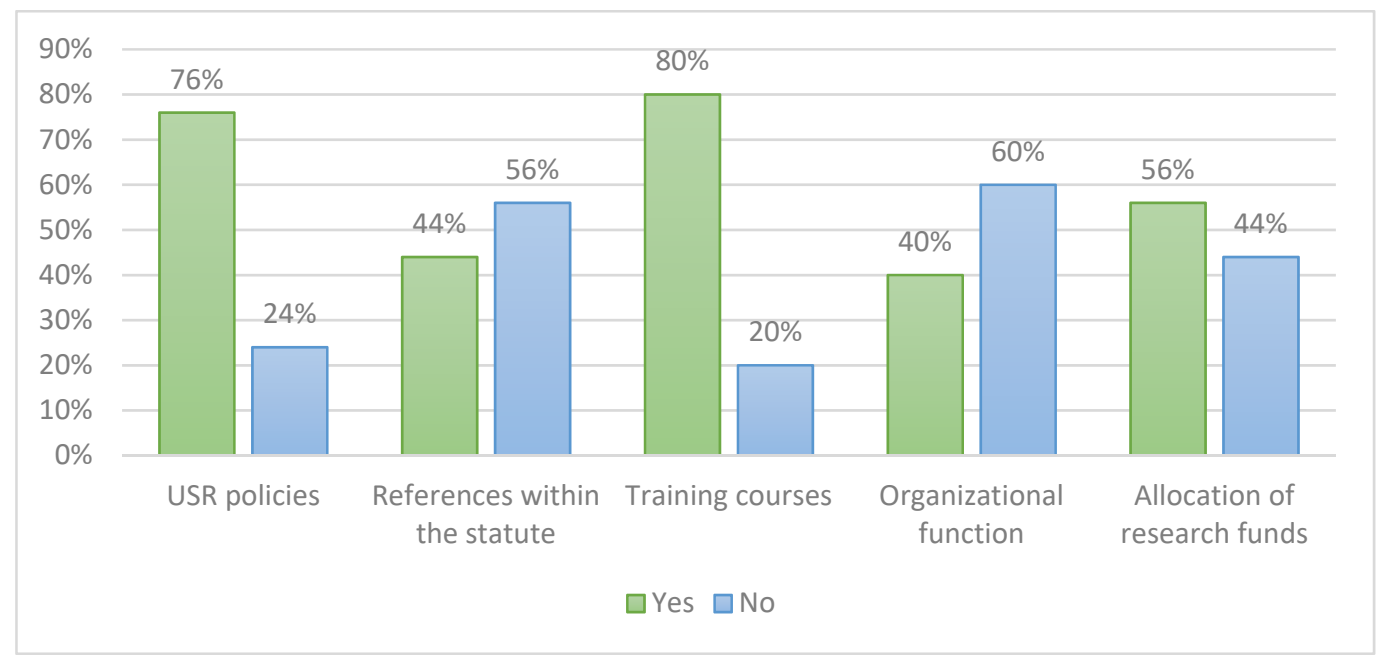

Figure 6. Results obtained in response to questions no. 1, 2, 3, 6, 7 (please refer to the questionnaire).

4.3.2. Membership of Organizations and Promotion of Information/Dissemination Activities

Most of the respondents show a good interest in the participation and sharing of sustainability practices: $84 \%$ of the respondents stated that they organize information and/or awareness events on issues relating to sustainable development, including in the academic field.

The responses provided highlight that their commitment is formally shared through conferences and testimonies, the promotion of round tables and seminar cycles, as well as through the presentation of books and articles.

There is also a sensitivity and commitment to the issue on the part of some spontaneously created student associations, which often offer concrete collaboration in the organization of events, also making their contribution during the presentation.

It would be interesting to investigate the presence or absence of internal student associations (formalized or not) elsewhere to understand the awareness of the student audience regarding the position of their university towards sustainability practices.

The participation in national and international associations/rankings and networks is significant (equal to $80 \%$ ), symptomatic of a desire for openness and confrontation with realities that are profoundly different from those that characterize the Italian panorama.

Always considerable, albeit with slightly lower percentages (76\%), is the implementation of a website dedicated to university sustainability policies (Figure 7).

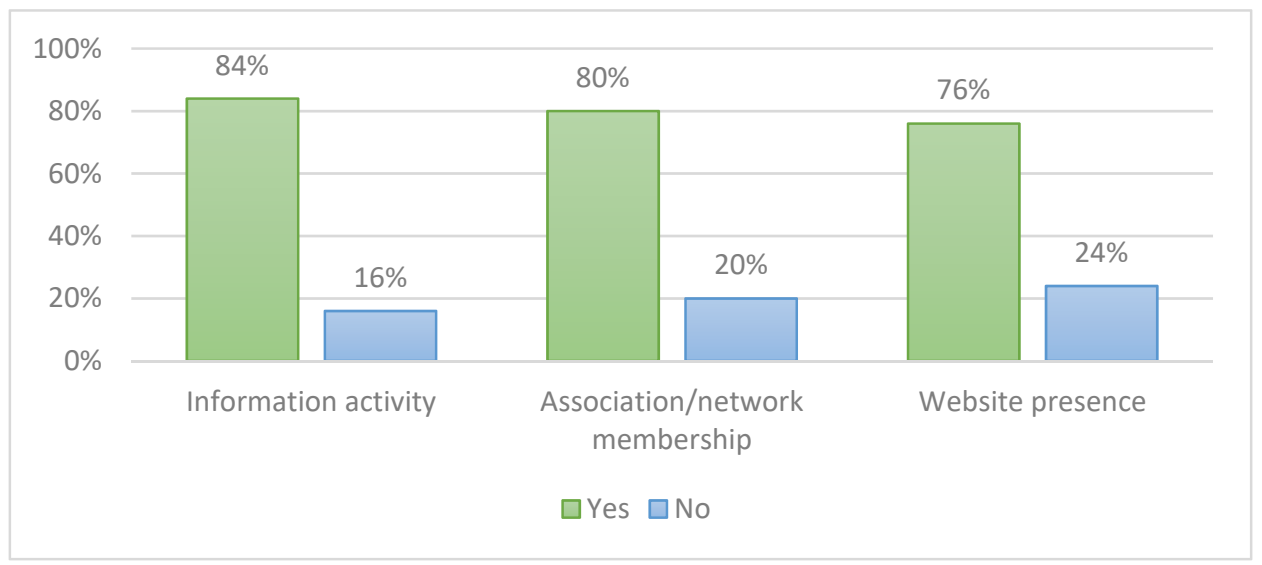

Figure 7. Results obtained in response to questions no. 4, 5, 8 (please refer to the questionnaire). 


\subsubsection{Actions in Favor of Sustainability}

This question aims to investigate what actions can be implemented to characterize a university as "sustainable".

A total of $84 \%$ of respondents are particularly attentive to issues relating to the efficiency of consumption related to the university structure (such as energy-saving and integrated management of the separate collection of waste produced).

With lower percentages, the themes related to:

- Dissemination of best practices both at a behavioral level (68\%) and within the individual teaching courses $(60 \%)$;

- Transport and mobility, through the promotion of sharing mobility policies and discounts on fares for the main public transport systems (52\%);

- Dematerialization of resources (compilation and submission of requests, registration of exams, management of practices relating to the secretariat, etc.) for a value equal to $48 \%$;

- Optimized management of food and non-food waste for universities that have campuses and internal canteens (equal to $44 \%$ ).

It is interesting to underline the three interventions indicated in the residual answer "other": while the first concerns the creation of living lab paths in which to experiment new techniques, behaviors and projects in favor of sustainability, the second and third answers focus on issues related to stakeholder engagement and the introduction of a social reporting document.

The latter, developed on the basis of indicators expressing the results achieved of an economic, social and environmental nature, is suitable for ensuring an adequate level of transparency towards stakeholders and a formalization of the commitments undertaken and the objectives to be achieved (Figure 8).

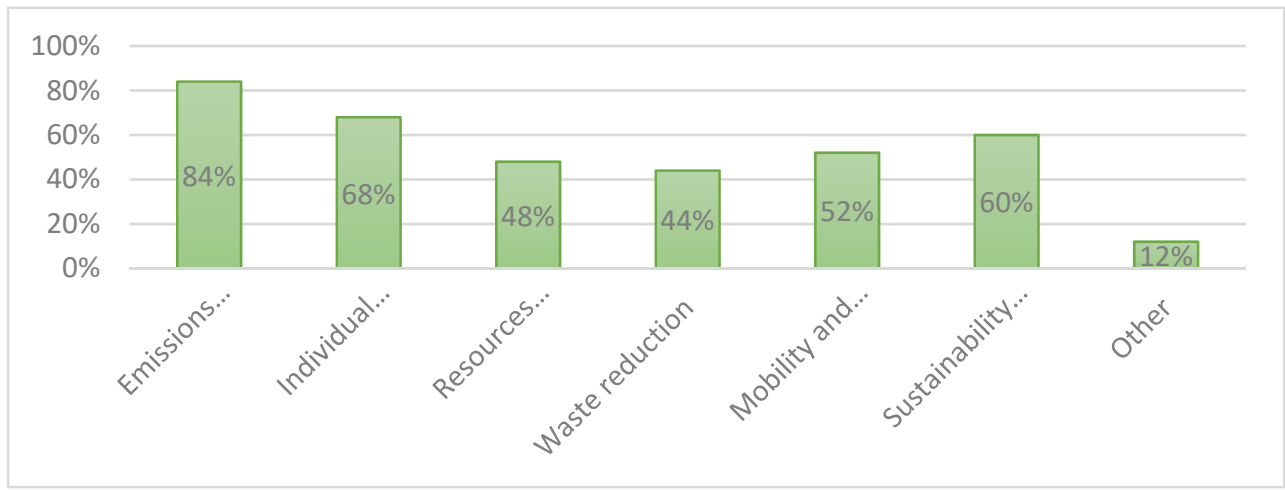

Figure 8. Result obtained in response to question no. 9 (please refer to the questionnaire).

It should be noted that the question administered is partially incomplete as it does not investigate the reasons and benefits underlying the implementation of USR policies (including social reporting) but rather seeks the actions that make a university sustainable. To fill this information gap, it was decided to go further into this topic during the interview in order to obtain information as complete and comprehensive as possible.

\subsubsection{Barriers Underlying the Adoption of USR Policies}

Concerning the constraints that hinder the adoption of USR policies and the implementation of a social reporting system, $80 \%$ of respondents argue that they are attributable to a lack of adequate resources and time they can dedicate. Or, if the university has these two factors, $60 \%$ say they find it difficult to find qualified people to follow this path.

The graph shows that $40 \%$ of respondents, if they had resources and time to dedicate, do not perceive the phenomenon as a priority element. On this issue, in the residual 
category "other" one respondent attributed the non-priority to a lack of interest on the part of the governing body in charge (Figure 9).

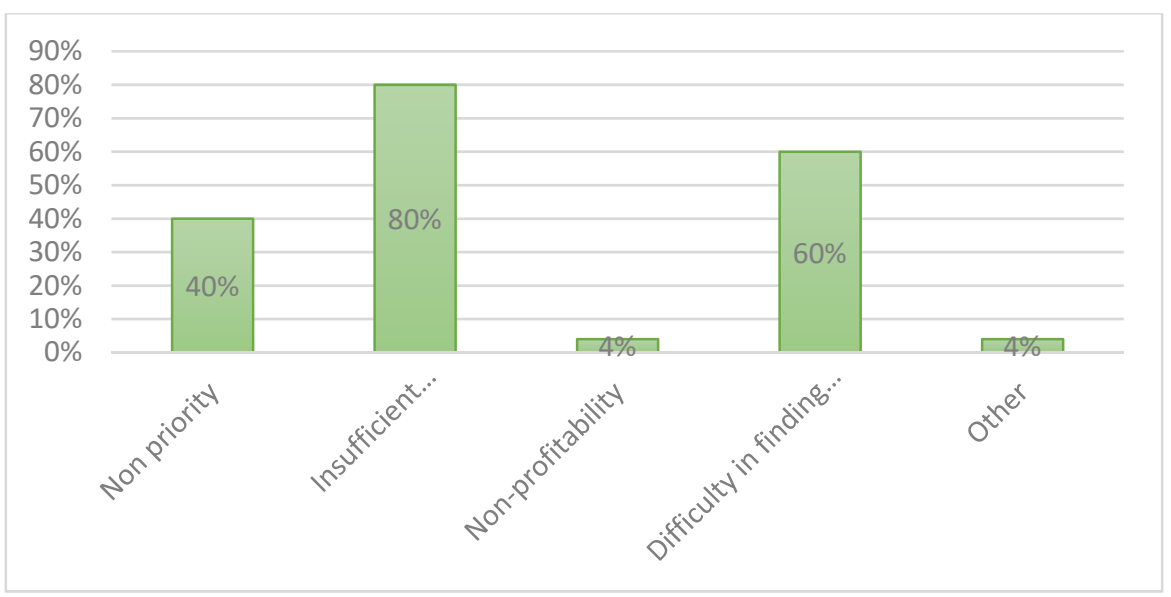

Figure 9. Result obtained in response to question no. 10 (please refer to the questionnaire).

In conclusion, only one respondent reported the non-profitability deriving from the implementation of sustainability policies and forms of social reporting. This statement could denote a lack of awareness of the potential of social reporting, and as Professor Moggi has stated, "this vision could be conveyed by short-term policies, which see social reporting as yet another accountability tool that would burden the already burdensome administrative commitments" (Moggi 2016).

\subsection{Interviews}

For a better understanding of the phenomenon, the results obtained by the four universities interviewed are presented below.

\subsubsection{The Theme of Social Reporting}

As this work path has shown, it can certainly be argued that the social reporting process impacts internal processes, governance and university performance and on the subjects participating in it (Moggi 2016).

The universities interviewed are also aware of this, regardless of the internal drafting of a formal document. Going into the merits of individual specificities, the non-reporting universities $\mathrm{B}$ and $\mathrm{C}$ demonstrate different approaches.

University B, although particularly sensitive to university social responsibility policies with particular reference to the theme of sustainable mobility and its diffusion within all levels of teaching, ascribes the different priorities to the non-adoption of social reporting on the part of the acting governing body as well as the lack of a formalized organizational structure that can guarantee constant commitment during the phases of the data processing and report drafting process.

University $C$ demonstrates a proactive interest in the subject, indicating how a proposal for the preparation of a draft sustainability report was included in the new strategic plan being approved. Addressing the causes of the delay in the implementation of the reporting compared to other universities, the interviewee stated that, despite sustainability initiatives already launched, it was decided to "delay" its publication in order to adapt the university policies, define suitable decision-making and operating procedures, as well as structuring its internal processes and information systems to be able to periodically collect and process data of an economic, social and environmental nature to be included in the report.

This "preparation" phase is preliminary to the creation of a programmatic tool, ensuring continuity of the project as well as adequate effectiveness and incisiveness towards its stakeholders regarding the activities carried out. By clarifying its intention to use the 
sustainability report as a "key to reading" for the definition, monitoring and evaluation of the strategies implemented over a long-term horizon, university $C$ is optimistic in stating how there is a virtuous path of convergence of "non-reporting" Italian universities about social reporting practices.

The positions and purposes of the two reporting universities A and D:

As regards university $\mathrm{A}$, the first (and only) version of the social report was published in the occasion of the tenth anniversary of the university's founding. Although further investigation and future developments were promoted in the text, it proved not to be not taken up in subsequent periods.

The drafted report set itself the objective of providing answers to a dual purpose: the first is to represent "a structured and synthetic tool for the evaluation of the results achieved", the second lies in the desire to promote "reflection and a comparison within the university "through the dissemination and sharing of information of a not strictly financial nature" functional to supplement (but not replace) the traditional reporting documents provided by the Ministry".

The work, carried out through the collaboration of a plurality of figures belonging to the faculty and the administrative-technical staff, took national and international guidelines as references for its drafting to represent the university in a more comprehensive and nonself-referential manner, stating its objectives, the use of the forms of financing received and the indication of the ample improvements "in view of a virtuous competition aimed at the overall improvement of the system". However, despite the hope of continuing this transparent and precise path of sharing and dialogue with the community and territory of reference, no subsequent versions have been proposed.

University D, with the publication of five social reports behind it, was selected to investigate the reasons behind their biennial publication and understand what prompted the university not to propose further versions.

Regarding the frequency of publication, since the objective of the social report is to systematize the relationship between the university and its community of reference as well as quantify the benefits in the area deriving from its presence (fully grasping the aspects of the third mission), not having to follow the typical frequency of financial statements, it was decided in the preliminary phase to follow a two-year frequency.

\subsubsection{Personal Perception of the Phenomenon}

As regards the personal perception of universities on the observed phenomenon, very different opinions were found depending on the basic culture, in particular regarding the interest on the part of the top academic staff.

Both universities $A$ and $B$ are aware of the value and potential of the report to ensure a better dialogue with their stakeholders and better monitoring and control of their strategic and operational objectives and understand that it is necessary to observe how to allocate the different action priorities of the governing bodies and the limited resources.

As regards university $\mathrm{A}$, despite having a background of knowledge and sensitivity towards carrying out this type of project, to date there is still no future prospect of further versions of the social report.

University B, in addition to the lack of interest on the part of the top management, does not yet have a formalized and structured organizational function to be able to follow a project of this magnitude.

University $C$ follows a different approach, seeing the budget as a tool for benchmarking the actions carried out from an economic, social and environmental point of view, underlining the importance of how the report should not be conceived as a mere addendum to the economic budget, but must be integrated into it by studying the destination and use of the forms of financing received, thus making it possible to fill the information gaps present within the documents provided for by the traditional reporting system. Among the barriers that have held adoption back is the absence of sufficient internal knowledge and skills of the academic institution, of the internal processes and policies implemented 
and the fact of not wanting to find external skills (as they do not possess the adequate sensitivity to create a reliable financial statement in the eyes of its stakeholders).

University D, by the particular geographical position in which it operates and the communicative nature of the tool, has introduced a social reporting system. Through the reporting of its actions and performance, the university can present itself to the local community, to the administration and institutions of the territory in order to be able to gain an ever-increasing amount of legitimacy and support. This commitment was formalized within the same reporting. Following the structure proposed by GBS, the University, since the penultimate edition, has aimed to add two new elements:

- The introduction of a section of a macroeconomic nature to highlight the weight and economic impact of the university on the reference area; through the use of macroeconomic indicators, the university has carried out a reporting analysis of the added value created, GDP and employment generated directly within the area and in relation to the related industries;

- The introduction of a gender report to provide an additional level of detail compared to that proposed by the GBS lines. this new path aims to constitute a first but important starting point in the definition of specific action aimed at ensuring greater transparency and efficiency of the university's work towards equal gender opportunities.

Regarding the barriers that hinder a complete implementation, as explained above, the main slowdown is represented by the heavy commitment to staff and structures in the data collection and processing phase. According to the interviewee, a further barrier to the creation of a social reporting system is represented by the priority variable: a university may decide not to implement any type of reporting form (but to use alternative forms of openness and collaboration) as it could be located within an economically more "prosperous" territory or may have established a consolidated relationship with it or even if there is no need to establish a strong bond.

\section{Discussion and Conclusions}

Following the disruptive processes of change at the national and international level, the formalization of the concepts of sustainability, social responsibility and the greater pressure exerted by the subjects who interface with the organizations, it was necessary, to respond to the new needs created, to introduce "alternative" reporting tools capable of overcoming the limits of the informative nature that characterize the typical tools of traditional reporting.

Originating initially within the for-profit sector, the issue of sustainability has also spread to the world of non-profit organizations and the public sector, which have had to and are still undergoing a process of change aimed at guaranteeing greater diffusion and sharing of the activities carried out and the consequent repercussions on the social, economic and environmental spheres.

First, through the analysis of the Italian academic landscape and the level of dissemination of social reporting and, subsequently, through the administration of questionnaires and interviews, the data analyzed as a whole were significant in illustrating the phenomenon being researched.

Specifically, the diachronic analysis, while outlining a path of change undertaken by a small number of universities, highlights strong elements of discontinuity in the reporting for the type of university, its size and its geographical positioning. In the decade 2008-2018, it is noted that only 38 out of 98 universities (38.78\%) published at least one form of social reporting, with the clarification that only three of these are non-state in nature.

It is assumed that there is little interest on the subject from private universities compared to those of a state nature, as the latter receives more external pressure regarding the reporting of public funds received as well as their use within the three missions. On the other hand, those of a non-state nature could be inspired by objectives aimed at highlighting the quality and effectiveness of the services offered, as well as incentives to raise additional funds and search for new donors. 
As far as geographical distribution is concerned, the reporting universities are mainly concentrated in the north (55.88\%) and the islands (50\%). Equally significant is the size of the university as most of the reporting universities have a size of more than 40,000 students with a reporting average of $83.33 \%$; a lower percentage large Universities are reporting $(57.89 \%)$ and medium with averages equal to $52.17 \%$; the presence of small reporting universities is much smaller, reaching a value of $13.89 \%$.

Regarding the consistency in the publication of reports, the survey highlighted a very fragmented situation, showing how often the first editions remain an isolated experiment or follow very inconsistent publications.

Given the objective difficulties in maintaining this "commitment", it is clear that this lack of consistency could have a negative impact on social interlocutors as they expect a continuous and constant "dialogue" with the university; this discontinuity could nullify the results obtained in the previous financial statements, undermining the programmatic capacity of this tool, as well as diminishing its effectiveness and incisiveness towards stakeholders.

In light of these conclusions, first by administering a questionnaire and subsequently by conducting interviews, an attempt was made to analyze in detail the aspects related to the personal perception of social reporting practices, the openness to university social responsibility policies and, through the selection of a sample of reporting and non-reporting universities, to investigate some peculiarities that emerged regarding the reasons that led them to adopt certain behavior and implement certain actions concerning social reporting.

The respondents confirmed the start of a virtuous process of change and greater openness to USR policies. Aware of how the university itself can represent an agent of change aimed at preserving the social, economic and environmental integrity of the current and future community, the respondents confirmed that the levers of teaching, information and dissemination of knowledge, research and membership of dedicated associations can represent useful tools for a better promotion and consolidation of sustainability.

However, as noted in the interview, those who are more open to the introduction of university sustainability practices or adhere to dedicated associations do not necessarily have social reporting tools in place. In fact, the universities being interviewed that currently do not report or report with discontinuities have shown themselves to be particularly attentive to these issues.

Although the driving force that allows the start of a social reporting process is often fueled and supported by the will and initiative of the teachers and research groups within the university, the project must necessarily be examined by the governing bodies so that its economic feasibility is assessed. Consequently, if the top management does not perceive a priority or guarantee its feasibility, there is the possibility that the project will not be carried out or that alternative forms of collaboration and openness will be opted for.

The last question focuses on the barriers that hinder a complete and continuous implementation of a social reporting system. From the answers obtained, the main limitations lie in the lack of human (qualified) and economic resources as well as a perception of the non-priority of the project by the universities.

Thanks to the interviews, this issue was explored with a greater level of detail, contextualizing it according to the university interviewed. More precisely, the interviews of the four case studies made it possible to highlight the realities in which the social reporting practice is already developed and others in which this practice is in the start-up phase (or has not yet been implemented), analyzing the personal perceptions of the motivations that promote their activities and related barriers.

At the end of this work, some observations can be made.

The first observation concerns the correlation between theoretical contribution and practical implications of our manuscript.

Via our survey some important theoretical points have come to the surface when addressing the topic of USR, in particular the size of the university, and the motivations and barriers underlying the implementation of a social reporting system. 
Those points assume a key role when assessing the practical implications of our work, since they depict the drivers towards a stronger (or a weaker) approach towards USR.

Assuming how a larger sample could have led to results that are more significant to explain the extent of the phenomenon, the main motivation that drives the university to implement social reporting tools lies in the demand to respond to two types of needs:

- The first, with internal value, proposes reports capable of overcoming the information limits present in traditional reporting by offering a programming, benchmarking and evaluation tool of the results achieved in the economic, social and environmental spheres;

- The second, of external nature, finds its existence in the involvement and reporting to the stakeholders of the path and the actions implemented by the university (guaranteeing greater legitimacy for the same to operate), verifying their effectiveness and consistency with its objectives and with its own mission.

However, aware of the potential that these tools can offer, there are some limits, particularly significant for small universities, which hinder their complete and continuous implementation. Apparently more facilitated in the data collection and processing phase thanks to the greater simplicity and flexibility of the organizational structure, this "advantage" could prove to be a weakness as, given the effort required in terms of time and resources, universities often do not have adequate organizational structures and qualified and available personnel to be able to carry out this type of path.

This first barrier, closely linked to the research process and implementation of a social reporting system, can be flanked by another, of a political-institutional nature: in fact, although the initiative is often undertaken by teachers and/or researchers, the governing bodies could block its progress both because they do not perceive reporting as a valuegenerating tool (consequently diverting the resources available to other issues) and because, despite the presence of interest, there are no structures and resources adequate to undertake and maintain the draft financial statements with continuity and reliability.

The paper is not without limitations.

As regards the questionnaire administration phase, the main critical elements include:

- e-Mail addresses of GBS contact persons found online not "updated" as the subjects indicated do not currently hold that role, consequently having to carry out further research to retrieve the current contact;

- A case in which a recipient replied as follows: "If you tell me what USR means, maybe I'll open the questionnaire. Cordially". This could lead to an inadequate knowledge base on the concept of university social responsibility: in this case, it was decided not to follow up on this contact as there would have been the possibility that the respondent did not have the adequate awareness to respond to the proposed questionnaire (except for the possibility of forwarding the same to a more competent person).

Here it is necessary to point out that the interview proved to be an extremely functional and decisive tool for the realization of the following work, allowing an adequate study of the topics partially addressed within the questionnaire.

Regarding the interviews proposed to the sample selected from the 98 universities, once the initial barriers of potential interviewees had been overcome in understanding the purposes of this study and the reasons that led to the selection of their own university, one of the main problems was found first and foremost in the difficulty in identifying the most suitable person to respond to the topics being interviewed (when not available directly through the RUS or GBS channels). This problem was accentuated in universities that pay little attention to issues related to social reporting, the spread of sustainability and social responsibility.

Secondly, despite the guarantee of anonymity, some interviewees proved reluctant to answer the question relating to the personal perception of the phenomenon, deciding not 
to expose themselves regarding the political choices of the university (about the reasons and the barriers underlying the implementation of a social reporting system).

Considering the difficulties indicated above, the significant dispersion and time delay (often with telephone "bounces" between the various organizational functions of the university) led to a consequent modification of the sample initially hypothesized.

Given the difficulties encountered and in the light of the sincere interest of some of the interviewees and respondents to the questionnaire, the aim of this paper was to contribute to research in the study of social reporting within the national academic panorama, in the hope that an increasing number of universities and institutions will fully understand the potential and validity of university social responsibility and social reporting practices, promoting further research on the subject and placing themselves as agents promoting policies aimed at more sustainable development.

Some reflections, finally, about the possible developments of the present work.

First, we would like to stress the importance of the theme of the USR in a broader logic, capable of valorizing the universities as factors and proactive agents of change.

At the same time, with specific reference to the Italian context, some critical points and elements limit its wider and more effective use, such as the absence of sufficient human and financial resources as well as technological equipment in line with the information needs of the USR.

Active fields of further development can be identified, then, both in an analysis of geographical contexts other than Italy, and in a temporal extension of the period of analysis, able to capture even the most recent academic years (from 2019 onwards).

In line with Nicolò et al. (2021) specific areas of information regarding USR, for instance: the impact of COVID-19 or the intellectual capital disclosure, could be assessed and as well as the different factors of sustainability practices (see for instance Larrán Jorge et al. 2019) could be investigated.

Furthermore, important insights could be fostered by GPP, for instance the positive role developed by external stakeholders' pressure.

At the same time, finally, the methodology adopted in this paper (questionnaire and semi-structured interviews) could be usefully enriched through other research mechanisms.

Author Contributions: Conceptualization, L.G., V.M., P.T. and M.C.; methodology and formal analysis, L.G. and V.M.; writing the paper L.G. and V.M. All authors have read and agreed to the published version of the manuscript.

Funding: This research received no external funding.

Institutional Review Board Statement: Not applicable.

Informed Consent Statement: Not applicable.

Data Availability Statement: The data presented in this study are available on request from the corresponding author.

Conflicts of Interest: The authors declare no conflict of interest. 


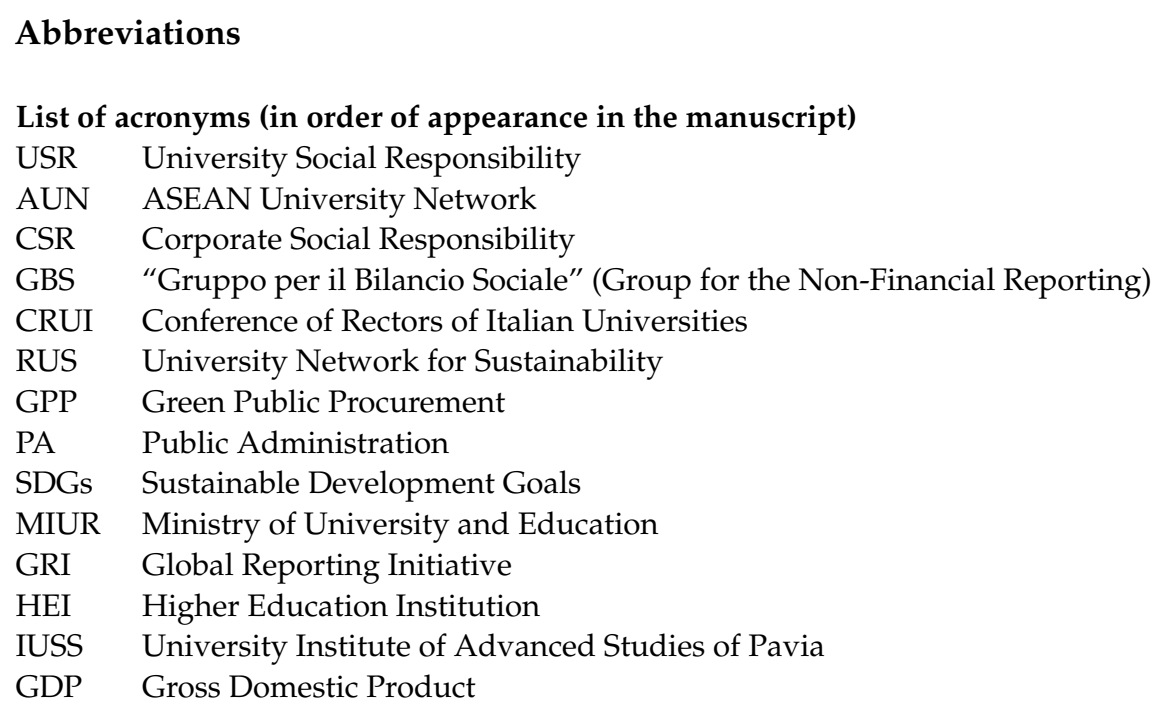

\section{Appendix A. The Survey}

1. Does the university adopt formalized policies (in terms of procedures and controls) in favor of University Social Responsibility (e.g., sustainability analysis of its suppliers, integration of the USR with the governance, living lab projects, involvement of its stakeholders in sustainability practices, etc.)?

- $\quad$ Yes

- No

- Other

2. Has the University included an explicit reference to the issue of sustainability in its Statute?

- $\quad$ Yes

- No

- Other

3. Does the university have ad hoc training courses on topics related to sustainability?

- $\quad$ Yes

- No

4. Does the university organize awareness-raising events on issues related to sustainable development, including in the academic environment (e.g., academic events, conferences, etc.)?

- Yes

- No

- Other

5. Does the university join, [...], to national and/or international associations/Ranking/ Networks?

- Yes

- No

- Other

6. Does the university allocate research funds to university sustainability?

- Yes

- No

7. Does the university have a specific organizational unit dedicated to university sustainability?

- Yes 
- No

8. Does the university have a website or a page within the university website dedicated to university sustainability policies?

- Yes

- No

9. What are the main challenges that the university should take up to be "more sustainable"?

- Reduction of emissions related to the university structure (reduction of energy consumption, waste, etc.)

- "Virtuous" individual behavior of university attendees (e.g., sustainable mobility, attention to waste disposal, etc.)

- Implementation of on-line services to reduce travel and resources (paper etc.)

- Reduction of food and non-food waste (energy, paper, etc.)

- Greater attention to issues related to the transport and mobility of students, professor, and technical-administrative staff

- Greater involvement and integration of the culture of sustainable development within the educational proposal

- Other

10. What does the university believe are the barriers to USR policies (including writing social reporting forms)?

- Perception of a non-priority for the university

- Insufficient resources/time to devote to the project

- Non-"profitability" of the project

- Difficulty in finding qualified personnel to carry out this type of project

- Other

\section{References}

Albrecht, Patrick, Simon Burandt, and Stefan Schaltegger. 2007. Do sustainability projects stimulate organizational learning in universities? International Journal of Sustainability in Higher Education 8: 403-15. [CrossRef]

Ali, Muhammad, Ts Ishamuddin Mustapha, Sharina Binti Osman, and Umar Hassan. 2020. University social responsibility (USR): An Evolution of the concept and its thematic analysis. Journal of Cleaner Production 286: 124931. [CrossRef]

ASEAN University Network (AUN). 2012. University Social Responsibility and Sustainability. Bangkok: AUN.

Bokhari, Abla A. H. 2017. Universities' Social Responsibility (USR) and Sustainable Development: A Conceptual Framework. SSRG International Journal of Economics and Management Studies 4: 8-16.

Buzby, Stephen L., and Haim Falk. 1979. Demand for Social Responsibility Information by University Investors. The Accounting Review 54: 23-37.

Cheng, Wenjuan, Andrea Appolloni, Alessio D'Amato, and Qinghua Zhu. 2018. Green Public Procurement, missing concepts and future trends-A critical review. Journal of Cleaner Production 176: 770-84. [CrossRef]

Cortese, Anthony D. 2003. The critical role of higher education in creating a sustainable future. Planning for Higher Education 31: 15-22.

del Mar Alonso-Almeida, Maria, Frederic Marimon, Fernando Casani, and Jesus Rodriguez-Pomeda. 2015. Diffusion of Sustainability Reporting in Universities: Current Situation and Future Perspectives. Journal of Cleaner Production 106: 144-54. [CrossRef]

Del Sordo, Carlotta, Federica Farneti, Silvia Pazzi, and Benedetta Siboni. 2010. Voluntary Reporting in Italian State Universities: Contents of social reports. Meditari Accountancy Research 24: 91-110.

Del Sordo, Carlotta, Federica Farneti, James Guthrie, Silvia Pazzi, and Benedetta Siboni. 2016. Social reports in Italian universities: Disclosures and preparers' perspective. Meditari Accountancy Research 24: 91-110. [CrossRef]

Fornasa, Walter, and Mario Salomone. 2007. Formazione e sostenibilità. Responsabilità sociale e culturale dell'università. Milano: Franco Angeli.

Fossati, Silvia, Laura Luoni, and Patrizia Tettamanzi. 2009. Il bilancio sociale e la comunicazione con gli stakeholder. Milano: Pearson.

Frey, Marco. 2009. Il bilancio sociale delle Università. Impresa Progetto-Electronic Journal of Management. 1. Available online: https:/ / www.impresaprogetto.it/en/essays/2009-1/ frey (accessed on 10 November 2020).

Galantino, Nunzio. 2017. La “Triplice Missione” dell’Università. Available online: https:/ /www.ilsole24ore.com/art/commenti-eidee/2017-04-07/la-triplice-missione-dell-universita-192554.shtml?uuid=AE8ghQ1 (accessed on 10 November 2020).

GBS. 2016. La rendicontazione sociale negli Atenei italiani: Valori, modelli e misurazioni. Milano: Franco Angeli. 
Gulbrandsen, Magnus, and Stig Slipersaeter. 2007. The third mission and the entrepreneurial university model. In Universities and Strategic Knowledge Creation. Specialization and Performance in Europe. Cheltenham: Edward Elgar Publishing.

Husband, Gary. 2020. Ethical Data Collection and Recognizing the Impact of Semi-Structured Interviews on Research Respondents. Education Sciences 10: 206. [CrossRef]

Jones, Paula, David Selby, and Stephen Sterling. 2010. Sustainability Education: Perspectives and Practice across Higher Education. London: Earthscan.

Larrán Jorge, Manuel, Francisco Javier Andrades Peña, and Jesús Herrera Madueño. 2019. Analysis of university sustainability reports from the GRI database: An examination of influential variables. Journal of Environmental Planning and Management 62: 1019-44. [CrossRef]

Lee, Yong S. 2000. The Sustainability of University-Industry Research Collaboration: An Empirical Assessment. The Journal of Technology Transfer 25: 111. [CrossRef]

Liu, Junqi, Yanlin Ma, Andrea Appolloni, and Wenjuan Cheng. 2021. How external stakeholders drive the green public procurement practice? An organizational learning perspective. Journal of Public Procurement 21: 138-66. [CrossRef]

Lozano, Rodrigo. 2011. The state of sustainability reporting in universities. International Journal of Sustainability in Higher Education 12: 67-78. [CrossRef]

Lozano, Rodrigo, Rebeka Lukman, Francisco J. Lozano, Donald Huisingh, and Wim Lambrechts. 2013. Declarations for sustainability in higher education: Becoming better leaders, through addressing the university system. Journal of Cleaner Production 48: 10-19. [CrossRef]

Ma, Yanlin, Yuting Liu, Andrea Appolloni, and Junqi Liu. 2021. Does green public procurement encourage firm's environmental certification practice? The mediation role of top management support. Corporate Social Responsibility and Environmental Management 28: 1002-17. [CrossRef]

Meseguer-Sánchez, Víctor, Emilio Abad-Segura, Luis Jesús Belmonte-Ureña, and Valentín Molina-Moreno. 2020. Examining the research evolution on the socio-economic and environmental dimensions on university social responsibility. International Journal of Environmental Research and Public Health 17: 4729. [CrossRef]

Moggi, Sara. 2016. Il sustainability reporting nelle università: Modelli, processi e motivazioni che inducono al cambiamento. Milano: Maggioli Editore.

Nicolò, Giuseppe, Natalia Aversano, Giuseppe Sannino, and Paolo Tartaglia Polcini. 2021. Investigating Web-Based Sustainability Reporting in Italian Public Universities in the Era of COVID-19. Sustainability 13: 3468. [CrossRef]

Rababah, Abedalqader, Natalya I. Nikitina, Veronica M. Grebennikova, Zhanna R. Gardanova, Angelina O. Zekiy, Vadim V. Ponkratov, and Nadezhda N. Bashkirova. 2021. University Social Responsibility during the COVID-19 Pandemic: Universities' Case in the BRICS Countries. Sustainability 13: 7035. [CrossRef]

Romolini, Alberto. 2015. Accountability e bilancio sociale negli enti locali. Milano: Franco Angeli.

RUS. 2019. Chi Siamo. Available online: https:/ / sites.google.com/unive.it/rus/chi-siamo?authuser=0 (accessed on 10 November 2020).

Rusconi, Gianfranco. 2005. Il bilancio sociale. Economia, etica e responsabilità dell'impresa. Roma: Ediesse.

Velazquez, Luis, Nora Munguia, Alberto Platt, and Jorge Taddei. 2006. Sustainable university: What can be the matter? Journal of Cleaner Production 14: 810-19. [CrossRef]

Wright, Tarah S. A. 2002. Definitions and frameworks for environmental sustainability in higher education. Higher Education Policy 15: 105-20. [CrossRef] 